\title{
Molecular Evidence for Antigen Drive in the Natural History of Mantle Cell Lymphoma
}

Aliki Xochelli, ${ }^{* \dagger \dagger}$ Lesley-Ann Sutton, ${ }^{\ddagger}$ Andreas Agathangelidis, ${ }^{\S}$ Evangelia Stalika, ${ }^{* \dagger}$ Maria Karypidou, ${ }^{* \dagger}$ Fotini Marantidou, ${ }^{\natural}$ Alba Navarro Lopez, Giorgos Papadopoulos, ${ }^{* *}$ Jana Supikova, ${ }^{\dagger \dagger}$ Patricia Groenen, ${ }^{\star *}$ Myriam Boudjogra, ${ }^{\$ \S}$ Christer Sundstrom, Maurilio Ponzoni, ${ }^{\top \uparrow}$ Hana Skuhrova Francova, ${ }^{\dagger \dagger}$ Achilles Anagnostopoulos, ${ }^{\dagger}$ Sarka Pospisilova, ${ }^{\dagger \dagger}$ Theodora Papadaki,,\|\| Dimitris Tzovaras, ** Paolo Ghia, ${ }^{\S}$ Christiane Pott, ** Frederic Davi, ${ }^{\S \S}$ Elias Campo, Richard Rosenquist, Anastasia Hadzidimitriou, ${ }^{*}$ Chrysoula Belessi, ${ }^{\mathbb{T}}$ and Kostas Stamatopoulos* ${ }^{* \ddagger}$

From the Institute of Applied Biosciences, * and the Information Technologies Institute,** CERTH, Center for Research and Technology Hellas, Thessaloniki, Greece; the Hematology Department and HCT Unit, ${ }^{\dagger}$ G. Papanicolaou Hospital, Thessaloniki, Greece; the Department of Immunology, Genetics and Pathology, ${ }^{\ddagger}$ Science for Life Laboratory, Uppsala University, Uppsala, Sweden; the Laboratory of B cell Neoplasia and Lymphoma Unit, ${ }^{\S}$ Division of Molecular Oncology and Department of Onco-Hematology, Università Vita-Salute San Raffaele, and the Pathology Unit and Unit of Lymphoid Malignancies, ${ }^{\top \uparrow}$ Istituto Scientifico San Raffaele, Milan, Italy; the Hematology Department, ${ }^{\rrbracket}$ Nikea General Hospital, Piraeus, Greece; the Insititut d'investigacions biomèdiques August Pi i Sunyer (IDIBAPS)," Hospital Clinic, University of Barcelona, Barcelona, Spain; the Department of Internal Medicine-Hematology and Oncology, ${ }^{\dagger \dagger}$ University Hospital Brno and Central European Institute of Technology, Masaryk University, Brno, Czech Republic; the Department of Pathology, ${ }^{\ddagger}$ Radboud University, Nijmegen Medical Centre, Nijmegen, the Netherlands; the Biological Hematology Service, Hopital PitieSalpetriere, ${ }^{\S \S}$ and UPMC Univ Paris 06, UMRS 1138, Paris, France; the Hematopathology Department, ${ }^{1 \| 1}$ Evangelismos Hospital, Athens, Greece; and the II. Medizinische Klinik und Poliklinik, *** University Hospital Schleswig-Holstein, Kiel, Germany

Accepted for publication February 26, 2015.

Address correspondence to Lesley-Ann Sutton, Ph.D., Department of Immunology, Genetics and Pathology, Science for Life Laboratory, Uppsala University, Dag Hammarskjolds vag 20, SE-751-85 Uppsala, Sweden. E-mail: lesley.sutton@ igp.uu.se.
To further our understanding about antigen involvement in mantle cell lymphoma (MCL), we analyzed the expression levels of activation-induced cytidine deaminase (AID), a key player in B-cell responses to antigen triggering, in $133 \mathrm{MCL}$ cases; assessed the functionality of AID by evaluating in vivo class switch recombination in $52 \mathrm{MCL}$ cases; and sought for indications of ongoing antigen interactions by exploring intraclonal diversification within $14 \mathrm{MCL}$ cases. The AID full-length transcript and the most frequent splice variants (AID- $\Delta E 4 a, A I D-\Delta E$ ) were detected in $128(96.2 \%), 96(72.2 \%)$, and 130 cases $(97.7 \%)$, respectively. Higher AID full-length transcript levels were significantly associated $(P<0.001)$ with lack of somatic hypermutation within the clonotypic immunoglobulin heavy variable (IGHV) genes. Median AID transcript levels were higher in lymph node material compared to cases in which peripheral blood was analyzed, implying that clonal behavior is influenced by the microenvironment. Switched tumor-derived IGHV-IGHD-IGHJ transcripts were identified in 5 of 52 cases $(9.6 \%)$, all of which displayed somatic hypermutation and AID-mRNA expression. Finally, although most cases exhibited low levels of intraclonal diversification, analysis of the mutational activity revealed a precise targeting of somatic hypermutation indicative of an active, ongoing interaction with antigen(s). Collectively, these findings strongly allude to antigen involvement in the natural history of MCL, further challenging the notion of antigen naivety. (Am J Pathol 2015, 185: 1740-1748; http://dx.doi.org/10.1016/j.ajpath.2015.02.006)
Supported in part by the ENosAI project (code 09SYN-13-880) cofunded by the EU and the Hellenic General Secretariat for Research and Technology (A.X., A.H. and K.S.); the GSRT KRIPIS action, funded by Greece and the European Regional Development Fund of the EU under the O.P. Competitiveness and Entrepreneurship, NSRF 2007-2013; the Swedish Cancer Society, the Swedish Research Council, Lion's Cancer Research Foundations in Uppsala (L-A.S. and R.R.); Associazione Italiana per la Ricerca sul Cancro (AIRC) (Investigator grant and Molecular Clinical Oncology Program 5xMille \#9965), Milano, Italy; Ricerca Finalizzata
2010, Ministero della Salute, Roma, Italy (P.Gh.); projects from MSMT CR CZ.1.05/1.1.00/02.0068 (CEITEC), SoMoPro project 3SGA5792, and IGA MZ CR NT13493-4/2012 (J.S., H.S.F. and S.P.); and the Spanish Ministerio de Economia y Competitividad Comisión Interministerial de Ciencia y Tecnología (CICYT) SAF12-38432; Institut National du Cancer (PAIR-Lymphomes; A.N.L. and E.C.). A.A. is the recipient of a fellowship from Associazione Italiana per la Ricerca sul Cancro AIRC (Triennial fellowship Guglielmina Lucatello é Gino Mazzega).

Disclosures: None declared. 
Affinity maturation of B-cell responses is critically dependent on diversification mechanisms induced by antigen encounter, namely, somatic hypermutation (SHM) and class switch recombination (CSR). ${ }^{1,2}$ Lying at the core of both SHM and CSR is activation-induced cytidine deaminase (AID), an enzyme normally expressed by activated $\mathrm{B}$ cells as a consequence of crosslinking of the B-cell receptor (BcR), CD40, surface receptors for IL-4, and Toll-like receptors (TLRs). ${ }^{1}$ Mounting evidence suggests that AID is a critical player in the immunobiology, not only of normal, but also of malignant, $\mathrm{B}$ cells, and a notable example is provided by chronic lymphocytic leukemia (CLL) where AID was shown to be fully functional and induced by mimicking the stimulation provided by $\mathrm{T}$ cells within the CLL microenvironment. ${ }^{3}$

Switching to a different B-cell malignancy, mantle cell lymphoma (MCL) represents $5 \%$ to $10 \%$ of non-Hodgkin lymphomas and generally exhibits an aggressive clinical behavior. ${ }^{4}$ Nevertheless, certain subsets of patients can follow a more favorable clinical course with prolonged periods of stable disease. ${ }^{5}$ Genetically, MCL is defined by the chromosomal translocation $\mathrm{t}(11 ; 14)(\mathrm{q} 13 ; \mathrm{q} 32)$ that juxtaposes the CCDN1 locus on chromosome 11 to the Ig heavy chain (IGH) locus located on chromosome 14. This aberration leads to constitutive overexpression of cyclin D1, and consequently, cell-cycle dysregulation. ${ }^{6}$

Until quite recently, the prevailing view was that MCL originates from a peripheral B cell located within the inner mantle zone, an area composed of naive pre-germinal center-type B cells. However, studies began to emerge that suggested a more complex ontogenetic model for this disease. ${ }^{7-12}$ Molecular evidence from our large immunogenetic analysis further supported this notion and cast serious doubts on the long-held belief that all MCL originated from antigen-inexperienced B cells. ${ }^{13}$ Indeed, we demonstrated remarkable biases in the Ig heavy variable (IGHV) gene repertoire of MCL and SHM within the rearranged IGHV genes of $70 \%$ of cases, ranging from minimal (in the majority of cases) to pronounced, ie, $<3 \%$ identity with germline sequences. ${ }^{13,14}$ These findings strongly support an antigen-driven origin for MCL, at least for a substantial fraction of the cohort. ${ }^{15}$

Further support to the notion that $\mathrm{BcR}$ signaling can be important for the onset and progression of MCL was provided by our subsequent study, which showed that MCL with mutated IGHV genes, SOX11 negativity, and non-nodal presentation corresponds to a disease subtype exhibiting more indolent behavior. ${ }^{16}$ This contrasts with classic MCL, which harbored unmutated IGHV genes, was SOX11 positive, and was genetically unstable as shown by the presence of more complex karyotypes. Leading on from this, the importance of immune signaling for lymphoma development and evolution has been highlighted by the identification of novel recurrent mutations in various signaling pathways that are critical for B cells. ${ }^{17}$ Finally, the importance of immune pathways in MCL is underscored by the clinical efficacy of pharmacological inhibitors of BcR signaling, eg, ibrutinib. ${ }^{18}$
In the present study, we sought to obtain additional molecular insight into the role of antigen interactions in MCL ontogeny and evolution. To this end, we first investigated AID mRNA isoform expression and isotype switch events in the largest-ever cohort studied for this purpose and subsequently looked for possible associations with SHM status and the tissue origin of the samples. In addition, we explored the timing of antigen interactions and whether MCL cells may continue to acquire hypermutations following leukemic transformation by examining intraclonal diversification (ID) within Ig genes in the context of ongoing SHM. We document AID mRNA expression in $>96 \%$ of cases, thus corroborating our hypothesis for antigen involvement in MCL ontogeny. The latter is also supported by our finding of a targeted ID process and CSR, thus alluding to antigen involvement throughout the life of at least a fraction of MCL cases.

\section{Materials and Methods}

\section{Study Population}

A total of 133 patients diagnosed with MCL, 10 patients with reactive lymph nodes but nonmalignant disorders, and 10 healthy controls were included in the study. The diagnosis of MCL was established according to the 2008 World Health Organization Classification criteria. ${ }^{19}$ The study was approved by the local ethics review committee of each institution.

\section{Materials}

Genomic DNA and/or total RNA were extracted as reported $^{7,10-13}$ from: i) patients with MCL: lymph nodes (LN; $n=79$ ), peripheral blood (PB; $n=44)$, spleen $(n=5)$, bone marrow $(n=3)$, or subcutaneous biopsies $(n=2)$; ii) patients with reactive LNs: LN biopsies, $n=$ 10 ; and iii) healthy individuals: $\mathrm{PB} \mathrm{CD} 19^{+}$cells from 10 healthy controls, negatively selected immediately after blood withdrawal using a B-lymphocyte enrichment kit (RosetteSep; StemCell Technologies, Vancouver, BC, Canada). The purity of all preparations was always $>99 \%$ (for $\mathrm{CD} 19^{+}$cells).

\section{PCR Amplification and Sequence Analysis of IGHV-IGHD-IGHJ Rearrangements}

PCR amplification and sequence analysis of IGHV-IGHDIGHJ rearrangements from patients with MCL was performed as reported. ${ }^{7,10-13,20,21}$

To evaluate the presence of ID, subcloning analysis of the IGHV-IGHD-IGHJ PCR amplicons from 14 MCL cases was performed, as previously reported. ${ }^{22}$ Sets of subcloned sequences obtained from the same sample were then assessed for ID by examination of sequence variation within the $\mathrm{V}$ domain following definitions previously proposed by our group. ${ }^{22-24}$ 


\section{Quantitative Real-Time PCR for AID Transcripts}

AID mRNA expression profiling was performed on PB, bone marrow, and LN samples from MCL patients and LN samples from patients with reactive lymphadenopathy. For MCL, the neoplastic lymphocytic infiltration within PB and bone marrow samples ranged from $52 \%$ to $98 \%$ (median $80 \%$ ); only 5 of 47 cases displayed $<70 \%$ clonal cells.

Three independent real-time fluorescence PCR (RQ-PCR) assays were used to quantitate the full-length AID transcripts (AID-FL) and the most frequent splice variants, AID- $\Delta \mathrm{E} 4 \mathrm{a}$ (lacking the first 30 nucleotides of exon 4) and AID- $\Delta \mathrm{E} 4$ (lacking the entire exon 4), as previously reported. ${ }^{25}$

More specifically, to produce plasmid DNA calibrators that contained the target gene sequences for each AID variant, we first amplified each transcript in three separate qualitative RQ-PCR reactions (Table 1). Each PCR product (single band) was gel purified (QIAquick Gel Extraction Kit; QIAGEN, Hilden, Germany), cloned into the pCR2.1 vector (TA Cloning Kit; Invitrogen, Paisley, UK), and sequenced (Beckman Coulter CEQ 8000 sequencer; Beckman Coulter, Fullerton, CA) to confirm the insert. Plasmids were extracted using the Midiprep kit (Invitrogen) and quantified spectrophotometrically with the copy number for $1 \mathrm{mg}$ estimated based on the molecular weight of the vector and the insert. Plasmids were serially diluted (10-fold dilutions from $10^{6}$ to $10^{1}$ copies per $5 \mu \mathrm{L}$ ), and standard curves for RQ-PCR were generated.

RQ-PCR reagents included $20 \mathrm{mmol} / \mathrm{L}$ Tris- $\mathrm{HCl}(\mathrm{pH}$ 8.4), $50 \mathrm{mmol} / \mathrm{L} \mathrm{KCl}, 4 \mathrm{mmol} / \mathrm{L} \mathrm{MgCl}_{2}, 200 \mu \mathrm{mol} / \mathrm{L} \mathrm{dATP}$, $200 \mu \mathrm{mol} / \mathrm{L} \mathrm{dCTP}, 200 \mu \mathrm{mol} / \mathrm{L}$ dGTP, $400 \mu \mathrm{mol} / \mathrm{L}$ dUTP, 0.5-U UDG, 0.75-U Platinum Taq DNA Polymerase, 400 $\mathrm{nmol} / \mathrm{L}$ concentration of each oligonucleotide primer, 200 $\mathrm{nmol} / \mathrm{L}$ concentration of each oligonucleotide probe, and $5 \mu \mathrm{L}$ of template RNA or standard in a final volume of $25 \mu \mathrm{L}$. All samples were run in duplicates according to the following protocol: initial incubation at $50^{\circ} \mathrm{C}$ for 2 minutes, 40 cycles of denaturation at $95^{\circ} \mathrm{C}$ for 15 seconds, annealing at $53^{\circ} \mathrm{C}$ for 15 seconds, and extension at $72^{\circ} \mathrm{C}$ for $15 \mathrm{sec}-$ onds. $A B L$ was used as a housekeeping gene using the FusionQuant Control Gene Standard kit (Ipsogen, Marseille, France) in an independent real-time PCR reaction, and all results were obtained as threshold cycle values.

AID-FL, AID- $\Delta$ E4a, AID- $\Delta E 4$, and ABL copy numbers were calculated using the appropriate standard curve. In each experiment, the cutoff value for positivity was set to 10 AID copies. The result for each AID transcript was expressed as AID copies per 10,000 copies ABL.

\section{CSR Transcripts}

Isotype-switched transcripts were investigated in $52 \mathrm{MCL}$ cases by RT-PCR and by using the IGHV subgroup-specific leader primer corresponding to the tumor-specific IGHVIGHD-IGHJ rearrangement in combination with the IGHC primers. Purified PCR products were directly sequenced on
Table 1 Nucleotide Sequence of Primers and Probes Used for RQ-PCR Amplification of AID mRNA Transcripts

\begin{tabular}{ll}
\hline $\begin{array}{l}\text { Oligonu- } \\
\text { cleotide }\end{array}$ & Sequence \\
\hline AID-1 & $5^{\prime}$-CAGGGAGGCAAGAAGACAC-3' \\
AID-2 & $5^{\prime}$-GCCTGATGGAAGAGTTTG-3' \\
AIDF & $5^{\prime}$-GAGGCAAGAAGACACTCTGG-3' \\
AIDR & $5^{\prime}$-GTGACATTCCTGGAAGTTGC-3' \\
AID-1F & $5^{\prime}$-TACTGCTGGAATACTTTTGTAGAA-3' \\
AID-2F & $5^{\prime}$-CCATCATGACCTTCAAAGAAAAC-3' \\
AID-3F & $5^{\prime}$-CCATCATGACCTTCAAAGCC-3' \\
AID-3Pro & $5^{\prime}$-FAM-ACGCATTTCGTACTTTGGGACTTTG- \\
& BHQ-3' \\
AID-1,2Pro & $5^{\prime}$-FAM-AAGAACTTTCAAAGCCTGGGAAGG- \\
& BHQ-3' \\
\hline
\end{tabular}

AID, activation-induced cytidine deaminase; RQ-PCR, real-time fluorescence PCR.

both strands, and each sequence was compared to the clonotypic IGHV-IGHD-IGHJ rearrangement of the same case to determine relatedness.

\section{Statistical Analysis}

Descriptive statistics for discrete parameters included counts and frequency distributions. For quantitative variables, statistical measures included means, medians, SD, and minimum-maximum values. The significance of bivariate relationships was assessed by $\chi^{2}$ and $t$-tests. For all comparisons, a significance level of $P$ at 0.05 was set, and all statistical analyses were performed with the statistical package, SPSS version 12.0 (SPSS, Chicago, IL).

\section{Results}

\section{Ig Gene Repertoire and SHM Analysis}

A productive IGHV-IGHD-IGHJ rearrangement was obtained for 127 of $133(95 \%)$ cases in our series. Consistent with previous reports, IGHV3-21 was the most frequently used gene (20.4\%), followed by IGHV4-34 (14.1\%), IGHV3-23 (11\%), and IGHVI-8 (7\%). ${ }^{7-13}$ Collectively, these four genes accounted for $>50 \%$ of our cohort (Figure 1).

Turning to SHM, all cases bearing even single nucleotide changes from the germline were considered as somatically hypermutated rather than adopting a $2 \%$ cutoff for germline identity. This latter cutoff largely stemmed from previous studies in CLL that found that assignment of IGHV-IGHDIGHJ gene rearrangements to the mutated IGHV or unmutated IGHV subset based on the $2 \%$ cutoff value for deviation from the closest IGHV germline gene discriminated between patients with a good or bad prognosis, respectively. ${ }^{26,27}$ However, from a biological perspective, this is an oversimplification, because a low mutational burden, even a single amino acid replacement, can be functionally relevant, as evidenced from studies on normal, autoreactive, and malignant B-cell clones. ${ }^{28}$ 
A

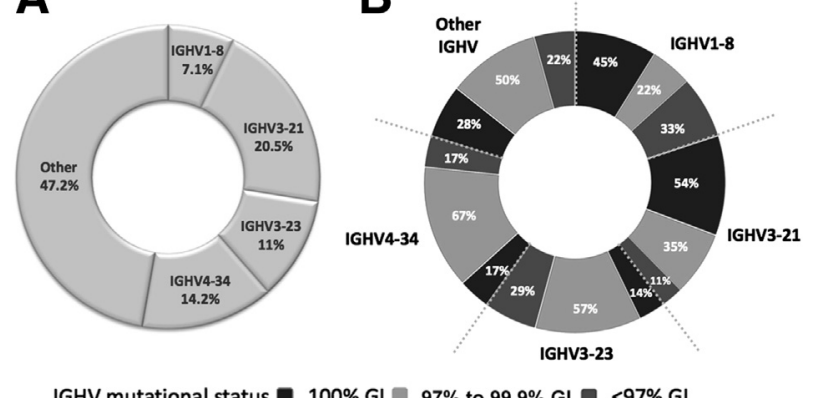

Figure 1 Immunoglobulin gene repertoire and level of somatic hypermutation within mantle cell lymphoma. A: The most frequently utilized IGHV genes in our cohort. Four IGHV genes represent $52.8 \%$ of the cohort analyzed. B: Rearrangements using each of these four genes display different profiles of somatic hypermutation. GI, germline identity.

Therefore, as in previous studies from our group, ${ }^{13,29}$ we adopted a different approach and separated the truly unmutated cases (100\% germline identity) from those cases exhibiting any level of SHM, and thus, considered them as mutated. Accordingly, only 40 of 127 rearrangements (31.5\%) carried IGHV genes with $100 \%$ identity to the closest germline gene (GI) and hence were assigned to the truly unmutated group. The remaining 87 of 127 cases (68.5\%) exhibited some impact of SHM ranging from minimal to pronounced. More specifically, following our previous definitions, ${ }^{13,14} 61$ of 127 cases (48\%) carried IGHV genes with $97 \%$ to $99.9 \%$ GI and hence were assigned to the borderline/minimally mutated group, whereas the remaining 26 of 127 cases $(20.5 \%)$ carried IGHV genes with $<97 \%$ GI and were considered as highly mutated. The cutoff of $3 \%$ is not used to disregard certain subgroups but rather to have a better and more detailed view of the mutational status within specific groups of patients such as those expressing a particular IGHV gene. Along these lines, of particular interest was the observation that rearrangements using certain IGHV genes displayed distinct SHM profiles, eg, the IGHV3-2I and IGHV4-34 genes were biased to either the truly unmutated or borderline/minimally mutated groups, respectively (Figure 1).

\section{The Clonotypic Ig Genes of MCL Can Be Intraclonally Diversified}

Fourteen MCL cases were analyzed for ID within their IGHV genes, resulting in a total of 331 subcloned sequences (14 to 47 per sample; median: 31 ). We intentionally evaluated cases that expressed one of the four most frequently used genes in MCL (IGHV3-21, $n=7$; IGHV4-34, $n=2$; IGHVI-8, $n=3$; IGHV3-23, $n=2$ ) (Supplemental Table $\mathrm{S} 1)$. Three cases were assigned to the truly unmutated category (100\% GI), seven cases were found to be borderline/ minimally mutated ( $97 \%$ to $99.9 \%$ GI), whereas the remaining four cases were highly mutated $(<97 \%)$.
Confirmed mutations (CMs), ie, mutations present in more than one but less than all subcloned sequences, and hence documenting ID, were discovered in 5 of 14 cases $(35.7 \%)$, whereas the remaining 9 cases $(64.3 \%)$ carried only unconfirmed mutations (UCMs), ie, mutations present in a single subcloned sequence (Table 2). The presence of mutations in the context of ID was independent of IGHV usage and/or the SHM status; CMs were observed in both minimally mutated (98\% to $99.9 \%$ GI) and highly mutated cases, whereas UCMs were evidenced across all three mutational groups (Table 2). Although a limited number of CMs per case was the general rule (four of five cases carrying CMs harbored only one to two CMs), examination of the UCMs that resulted in an amino acid change (one to six per sample; median: 3) provided further evidence for the very precise targeting of mutations introduced as part of the ID process. More specifically, some amino acid changes identified in single subcloned sequences were shared by all subcloned sequences of another case using the same IGHV gene, therefore alluding to a functionally driven response to an antigenic stimuli rather than a random event (Table 3). Consequently, several UCMs could justifiably be considered confirmed by another case.

Despite the overall low level of ID observed, a single case expressing a mutated IGHV3-21 BcR exhibited extensive ID with $21 \mathrm{CMs}$ at the amino acid level. The pronounced impact of ID evidenced in this case could be attributed to distinct clusters of subcloned sequences with cluster-specific mutational profiles. Analysis of the SHM patterns revealed that all clusters carried shared mutations and closely similar, if not identical, VH CDR3s (including identical length), pointing to the emergence from a common ancestor. The early branching of the leukemic clone in this case may

Table 2 Intraclonal Diversification Analysis within the IGHV Genes of 14 MCL Cases

\begin{tabular}{lrlrrrll}
\hline Case & CM & UCM & R & S & $\begin{array}{l}\text { AA } \\
\text { CM- }\end{array}$ & $\begin{array}{l}\text { AA } \\
\text { UCM- }\end{array}$ & $\begin{array}{l}\text { No. subcloned } \\
\text { sequences } \\
\text { analyzed }\end{array}$ \\
\hline BR18 & 1 & 2 & 0 & 1 & 0 & 2 & 19 \\
BR25 & 0 & 4 & 0 & 0 & 0 & 4 & 14 \\
10219 S & 2 & 4 & 1 & 1 & 2 & 3 & 38 \\
7591ST & 0 & 2 & 0 & 0 & 0 & 2 & 24 \\
M5 & 0 & 5 & 0 & 0 & 0 & 3 & 22 \\
M10 & 0 & 3 & 0 & 0 & 0 & 1 & 19 \\
M14 & 0 & 7 & 0 & 0 & 0 & 5 & 24 \\
B7 & 38 & 2 & 21 & 17 & 18 & 2 & 47 \\
B4 & 0 & 5 & 0 & 0 & 0 & 3 & 24 \\
B15 & 1 & 9 & 1 & 0 & 1 & 5 & 15 \\
7761ST & 0 & 4 & 0 & 0 & 0 & 2 & 21 \\
7836ST & 0 & 9 & 0 & 0 & 0 & 6 & 35 \\
BR14 & 0 & 4 & 0 & 0 & 0 & 3 & 14 \\
BR15 & 1 & 2 & 0 & 1 & 0 & 1 & 15 \\
\hline
\end{tabular}

$A A$, amino acid; $C M$, confirmed mutation; ID, intraclonal diversification; $M C L$, mantle cell lymphoma; $R$, replacement; $S$, silent; UCM, unconfirmed mutation. 
Table 3 Restricted Patterns of Intraclonal Diversification and Precise Targeting of Mutations

\begin{tabular}{|c|c|c|c|c|c|c|c|c|c|}
\hline Case & $\begin{array}{l}\text { IGHV } \\
\text { gene }\end{array}$ & Region & Codon & Germline AA & $\begin{array}{l}\text { IMGT } \\
\text { physicochemical } \\
\text { class }\end{array}$ & $\begin{array}{l}\mathrm{AA} \\
\text { replacement }\end{array}$ & $\begin{array}{l}\text { IMGT } \\
\text { physicochemical } \\
\text { class }\end{array}$ & $\begin{array}{l}\text { AA } \\
\text { change }\end{array}$ & $\begin{array}{l}\text { Mutation } \\
\text { category: } \\
\text { CM/UCM/shared }\end{array}$ \\
\hline $7591 S$ & $3-21 * 01$ & FR2 & 36 & $S$ & Polar, noncharged & $\mathrm{T}$ & Polar, noncharged & $\mathrm{S} \rightarrow \mathrm{T}$ & Shared \\
\hline B7 & $3-21 * 01$ & FR2 & 36 & $S$ & Polar, noncharged & $\mathrm{N}$ & Polar, noncharged & $\mathrm{S} \rightarrow \mathrm{N}$ & CM \\
\hline 7761 & $3-23 * 01$ & FR2 & 36 & $S$ & Polar, noncharged & $\mathrm{N}$ & Polar, noncharged & $\mathrm{S} \rightarrow \mathrm{N}$ & Shared \\
\hline $7591 S$ & $3-21 * 01$ & CDR2 & 63 & $S$ & Polar, noncharged & G & Nonpolar aliphatic & $\mathrm{S} \rightarrow \mathrm{G}$ & Shared \\
\hline M14 & $3-21 * 01$ & CDR2 & 63 & $S$ & Polar, noncharged & G & Nonpolar aliphatic & $\mathrm{S} \rightarrow \mathrm{G}$ & UCM \\
\hline 10,219 & $1-8 * 01$ & CDR2 & 64 & $\mathrm{~N}$ & Polar, noncharged & D & Acidic & $\mathrm{N} \rightarrow \mathrm{D}$ & Shared \\
\hline BR18 & $1-8 * 01$ & CDR2 & 64 & $\mathrm{~N}$ & Polar, noncharged & D & Acidic & $\mathrm{N} \rightarrow \mathrm{D}$ & UCM \\
\hline M10 & $3-21 * 01$ & FR3 & 72 & K & Basic & $\mathrm{R}$ & Basic & $\mathrm{K} \rightarrow \mathrm{R}$ & Shared \\
\hline
\end{tabular}

Precise targeting of intraclonal diversification infers strong functional constraints for preservation of critical physicochemical properties. Amino acids are represented by a single-letter code.

$A A$, amino acid; CDR, complementarity-determining region; CM, confirmed mutation; FR, framework region; IMGT, International ImMunoGeneTics Information System; UCM, unconfirmed mutation.

indicate that distinct subclones evolved along similar, although separate pathways, perhaps as a result of selective pressures occurring in parallel in distinct subclones, thereby fine-tuning their BcR affinities (Figure 2 and Supplemental Table S2).

\section{AID mRNA Is Detected in MCL, with Higher Expression in LNs Compared to PB}

AID mRNA expression was studied by RQ-PCR for the full-length transcript (AID-FL) and the most frequent splice variants, AID- $\Delta \mathrm{E} 4 \mathrm{a}$ and AID- $\Delta \mathrm{E} 4$. Results for each AID transcript were expressed as the percentage of AID copies per ABL copies.
All MCL cases expressed at least one AID transcript, with the vast majority expressing the AID-FL transcript (128 of 133 cases, $96.2 \%$ ) and the AID- $\Delta \mathrm{E} 4$ splice variant (130 of 133 cases; $97.7 \%$ ); the AID- $\Delta \mathrm{E} 4 \mathrm{a}$ transcript was detected in 96 of $133(72.2 \%)$ cases. Significant differences were reported in AID mRNA expression between different MCL cases. In fact, AID-FL transcript levels differed between MCL cases by up to 5-log, AID- $\Delta$ E4a splice variant up to $2-\log$, and AID- $\Delta E 4$ splice variant up to $4-\log$. AID transcripts were detected in all five MCL cases that were found to display ID within their Ig genes.

Given the difference in tissue origin of our sample collection, we considered samples derived from PB separately from those obtained from LN biopsies. All other tissue sources were excluded from comparison studies because

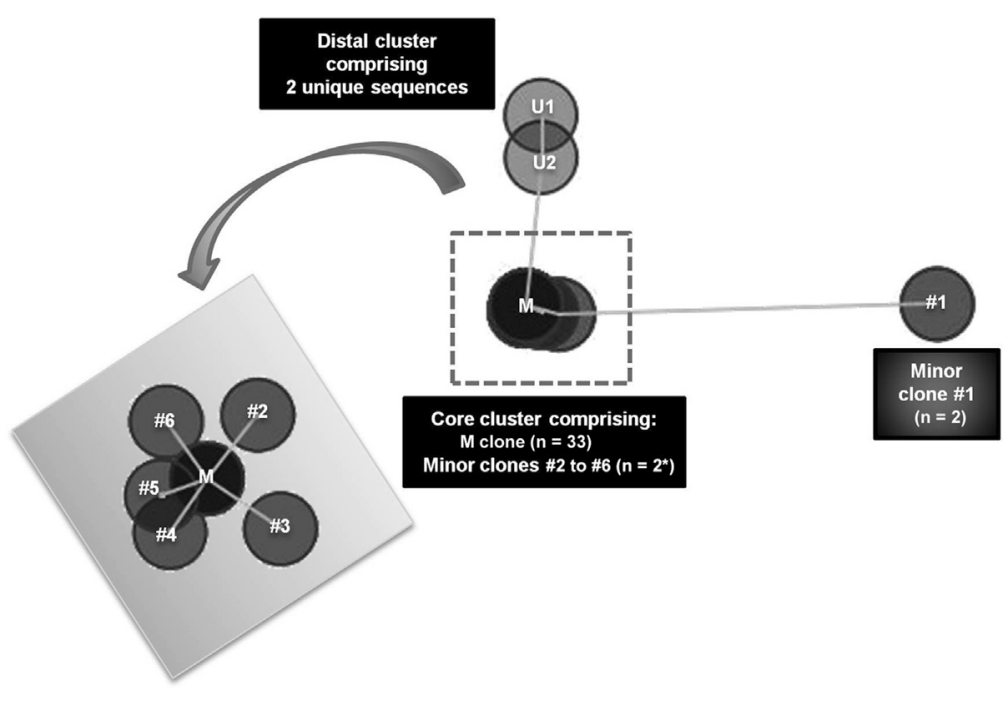

Figure 2 Intraclonal diversification can be a feature of mantle cell lymphoma (MCL). Cluster formation following analysis of IGHV-IGHD-IGHJ amino acid subcloned sequences from an MCL patient exhibiting intraclonal diversification. Three distinct clusters are observed. A core cluster was created that contained the major clone $(\mathrm{M})$, comprising 33 identical subcloned sequences, and five minor clones (\#2 to \#6), each consisting of two identical subcloned sequences (all minor clones differed from the major clone by only a single amino acid). From this core, two clusters further radiated: a minor clone comprised of two identical subcloned sequences, which varied considerably from the major clone, hence its distant location, but did share two amino acid replacements, thereby implying derivation from a common ancestor; and a distal cluster composed of two unique sequences (U1 and U2), which differed from each other by only one amino acid. The core cluster is highlighted by the dotted lines, and a more detailed view of this cluster is provided in the shaded gray box. Details of individual amino acid changes are provided in Table 3 and Supplemental Table S2. $n=47$ IGHV-IGHD-IGHJ amino acid subcloned sequences. *Each minor clone contains two identical sequences. 


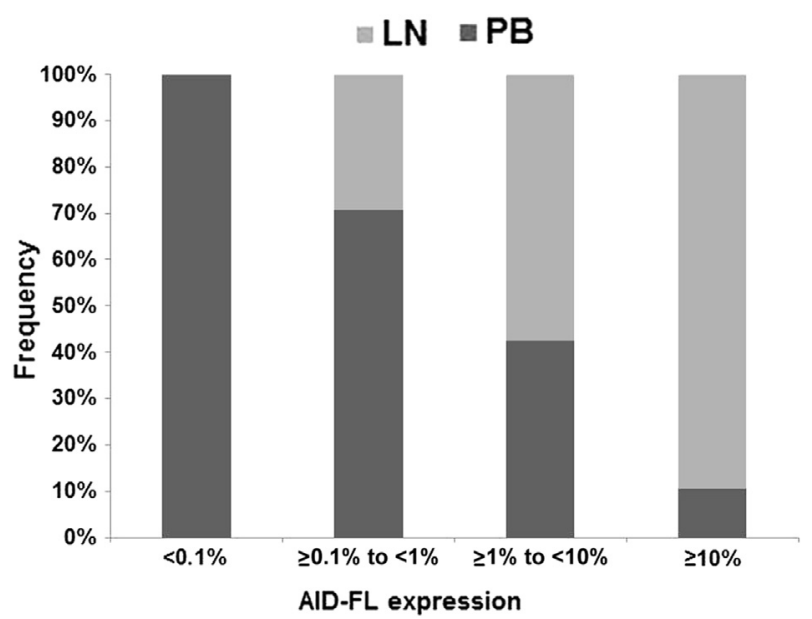

Figure 3 Activation-induced cytidine deaminase (AID) mRNA expression within different tissue types. Lymph nodes (LNs) were found to express higher levels of AID full-length (AID-FL) mRNA transcript levels in comparison to peripheral blood (PB), suggesting distinct microenvironmental effects in different tissue compartments. The horizontal axis refers to AID/ $A B L$ copy ratios.

of the small number of cases. This analysis revealed that $\mathrm{LN}$ samples expressed significantly $(P<0.0001)$ higher AIDFL transcript levels compared to PB samples (Figure 3). AID mRNA levels in LN samples from patients with MCL or patients with reactive lymphadenopathy $(n=10)$ did not differ significantly $(P=0.2)$. By contrast, all $\mathrm{CD} 19^{+}$cell preparations from 10 healthy individuals were found to be negative for AID mRNA expression.

Interestingly, in both $\mathrm{PB}$ and $\mathrm{LN}$ samples from patients with MCL, AID mRNA levels were higher in cases expressing unmutated IGHV genes. In fact, a highly significant $(P<0.001)$ association was noted between AID-FL transcript levels and IGHV genes with no SHM or borderline/minimally IGHV-mutated status: in particular, 35 of $40(87.5 \%)$ cases carrying IGHV genes with $100 \%$ GI expressed high AID-FL transcript levels compared to 46 of $61(75.4 \%)$ cases with $97 \%$ to $99.9 \%$ IGHV GI and only 11 of $26(42.3 \%)$ cases with $<97 \%$ IGHV GI (Figure 4).

\section{CSR Is a Feature of MCL}

Evidence that ongoing CSR may occur in MCL has been reported independently by several groups ${ }^{30-32}$ however the studied series were small, thus hindering definitive conclusions. We herein investigated isotype switch events in 52 MCL cases, all expressing mu and/or delta heavy chain isotypes, by flow cytometry and/or immunohistochemistry. Within this group, 36 cases (69.2\%) expressed highly or minimally/borderline mutated IGHV genes, whereas the remaining 16 cases $(30.8 \%)$ expressed truly unmutated IGHV genes. Overall, 5 of 52 cases $(9.6 \%)$ were found to carry alternative tumor-derived gamma $(n=1)$ or alpha $(n=4)$ transcripts; all 5 cases showed some impact of SHM, with 3 of 5 cases being assigned to the highly mutated group. All 5 cases expressed AID mRNA transcripts whereas 2 of 5 expressed high AID-FL transcript levels $(>1 \%)$.

\section{Discussion}

Neoplastic B cells retain the ability to home to tissue compartments where they can receive critical prosurvival and/or proliferation signals, including triggering through the $\mathrm{BcR}^{33}$ and other immune receptors, eg, the TLRs. ${ }^{34,35}$ This has set the ground for the development of alternative lymphoma treatments that target the surrounding tissue microenvironment and/or inhibit immune signaling, and they have already shown promising clinical activity in various B-cell lymphomas, including MCL. Indeed, the Bruton's tyrosine kinase inhibitor, ibrutinib, was recently found to display durable single-agent activity in relapsed and refractory MCL, ${ }^{18}$ providing perhaps the strongest proof of concept that microenvironmental drive is implicated in MCL ontogeny and evolution, as previously reported. ${ }^{13,14}$

From an immunological perspective, our present cohort can be considered as representative of the MCL population in general, based on the Ig gene repertoire and SHM status of the investigated cases. The highly restricted Ig gene repertoire of MCL, along with the observation that certain IGHV genes display distinctive SHM profiles, hints to functionally driven selective pressures being exerted on MCL progenitors that express distinct germline-encoded $\mathrm{VH}$ specificities, thus emphasizing the role of antigen involvement in MCL ontogeny. ${ }^{7-13}$

Although direct proof is not yet available, it can be argued that AID is likely functional and relevant in MCL

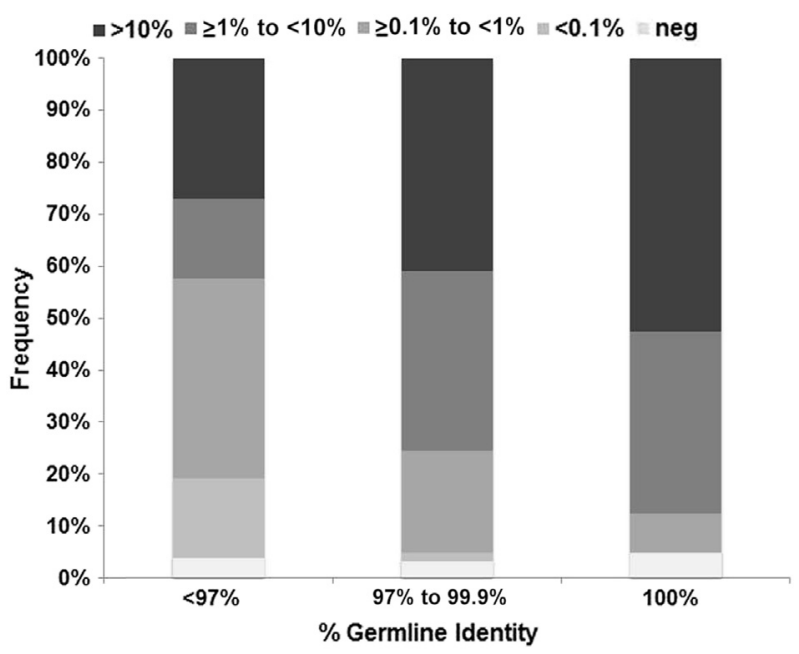

Figure 4 Activation-induced cytidine deaminase (AID) mRNA expression and IG mutational status. The expression level of the AID full-length (AID-FL) transcript varies depending on the level of somatic hypermutation, ie, AID mRNA levels are higher in cases expressing unmutated IGHV genes. The horizontal axis refers to the percent germline identity for the IGHV gene, whereas the shading indicates the various AID/ABL copy ratios. neg, negative. 
because most malignant clones carry BcR Igs with imprints of SHM and, as evidenced by this study, may also display ongoing isotype switch events and ID. The fact that different tissues express varying levels of AID isoform mRNA indicates that the microenvironment may be a critical factor for shaping clonal behavior and implies that malignant $\mathrm{B}$ cells may behave differently depending on their location. ${ }^{36}$ Examination of AID mRNA isoform expression within different tissue specimens (preferably LN and PB) from the same patient and time point would be highly informative; however, such samples were lacking. In addition, it should be taken into account that the analysis was conducted on whole-tissue samples and not on purified tumor cells. However, results were consistent between cases and, more importantly, can be safely considered as representative of the tumor population because the diagnostic LN samples showed complete effacement as a result of diffuse lymphomatous infiltration.

Although the exact function, if any, of the various AID isoforms remains unclear, ${ }^{37}$ some studies suggest that the alternative AID splice variants display different activities ranging from inactivation of CSR and SHM activity or, alternatively, to heightened SHM activity. ${ }^{38}$ By contrast, other studies report all alternative splice variants as nonfunctional, ${ }^{39}$ hence leaving the issue still under debate. We found that AID$\triangle \mathrm{E} 4 \mathrm{a} / \mathrm{ABL}$ and $\mathrm{AID}-\Delta \mathrm{E} 4 / \mathrm{ABL}$ transcript levels were much lower than those of the native, full-length AID mRNA, perhaps suggesting that splice variants may play a limited role, if any, in MCL physiology. Nevertheless, our finding of AID mRNA expression in $>96 \%$ of MCL cases strongly alludes to AID involvement in the natural history of MCL and bears resemblance to what has been shown for other B-cell malignancies, most notably CLL. $3,25,40-42$

A major analogy to CLL also concerns the association between AID mRNA levels and IGHV-unmutated status. Within the CLL setting, this association has been interpreted in the context of a more activated status and linked to a more aggressive disease, indicating intense microenvironmental triggering. ${ }^{3}$ In MCL, such evidence is still lacking, and the types of stimuli inducing AID expression, as well as the exact timing and location of these interactions, are yet to be defined. However, based on our results, it is tempting to argue that, similar to CLL, MCL cells are more intensely triggered within the tissue microenvironments than the circulation, underlying the observed higher AID expression in LNs compared to PB.

In conclusion, the above findings add further support to our previous claim that MCL ontogeny is antigendriven. ${ }^{13,14}$ In particular, we identified AID expression in the vast majority of MCL, with significantly higher levels among IGHV-unmutated cases, and also found in vivo CSR events in a proportion of the cases analyzed. These features along with the highly distinctive Ig gene repertoire observed within MCL, the functionally driven SHM, and our finding of a targeted ID process strongly indicate a role for antigen(s) in MCL development and also open possibilities for future investigations into the ontogeny of MCL.

\section{Acknowledgments}

A.X., L.A.S., and A.Ag. performed research, analyzed data, and wrote the manuscript; E.S., M.K., F.M., A.N.L., G.P., J.S., M.B., M.P., and H.S.F. performed research and analyzed data; C.S. and A.An. contributed samples and interpreted data; P.G., S.P., T.P., D.T., P.G., C.P., F.D., E.C., R.R., and A.H. supervised research and interpreted data; C.B. and K.S. designed the study, supervised research, and wrote the manuscript.

\section{Supplemental Data}

Supplemental material for this article can be found at http://dx.doi.org/10.1016/j.ajpath.2015.02.006.

\section{References}

1. Di Noia JM, Neuberger MS: Molecular mechanisms of antibody somatic hypermutation. Annu Rev Biochem 2007, 76:1-22

2. Chaudhuri J, Basu U, Zarrin A, Yan C, Franco S, Perlot T, Vuong B, Wang J, Phan RT, Datta A, Manis J, Alt FW: Evolution of the immunoglobulin heavy chain class switch recombination mechanism. Adv Immunol 2007, 94:157-214

3. Patten PE, Chu CC, Albesiano E, Damle RN, Yan XJ, Kim D, Zhang L, Magli AR, Barrientos J, Kolitz JE, Allen SL, Rai KR, Roa S, Mongini PK, MacCarthy T, Scharff MD, Chiorazzi N: IGHVunmutated and IGHV-mutated chronic lymphocytic leukemia cells produce activation-induced deaminase protein with a full range of biologic functions. Blood 2012, 120:4802-4811

4. Vose JM: Mantle cell lymphoma: 2012 update on diagnosis, riskstratification, and clinical management. Am J Hematol 2012, 87: 604-609

5. Royo C, Navarro A, Clot G, Salaverria I, Gine E, Jares P, Colomer D, Wiestner A, Wilson WH, Vegliante MC, Fernandez V, Hartmann EM, Trim N, Erber WN, Swerdlow SH, Klapper W, Dyer MJ, Vargas-Pabon M, Ott G, Rosenwald A, Siebert R, Lopez-Guillermo A, Campo E, Bea S: Non-nodal type of mantle cell lymphoma is a specific biological and clinical subgroup of the disease. Leukemia 2012, 26 : 1895-1898

6. Jares P, Colomer D, Campo E: Molecular pathogenesis of mantle cell lymphoma. J Clin Invest 2012, 122:3416-3423

7. Camacho FI, Algara P, Rodriguez A, Ruiz-Ballesteros E, Mollejo M, Martinez N, Martinez-Climent JA, Gonzalez M, Mateo M, Caleo A, Sanchez-Beato M, Menarguez J, Garcia-Conde J, Sole F, Campo E, Piris MA: Molecular heterogeneity in MCL defined by the use of specific VH genes and the frequency of somatic mutations. Blood 2003, 101:4042-4046

8. Kienle D, Krober A, Katzenberger T, Ott G, Leupolt E, Barth TF Moller P, Benner A, Habermann A, Muller-Hermelink HK, Bentz M, Lichter P, Dohner H, Stilgenbauer S: VH mutation status and VDJ rearrangement structure in mantle cell lymphoma: correlation with genomic aberrations, clinical characteristics, and outcome. Blood 2003, 102:3003-3009

9. Orchard J, Garand R, Davis Z, Babbage G, Sahota S, Matutes E, Catovsky D, Thomas PW, Avet-Loiseau H, Oscier D: A subset of t(11; 14) lymphoma with mantle cell features displays mutated $\mathrm{IgVH}$ genes and includes patients with good prognosis, nonnodal disease. Blood 2003, 101:4975-4981 
10. Schraders M, Oeschger S, Kluin PM, Hebeda K, Schuuring E, Groenen PJ, Hansmann ML, van Krieken JH: Hypermutation in mantle cell lymphoma does not indicate a clinical or biological subentity. Mod Pathol 2009, 22:416-425

11. Thorselius M, Walsh S, Eriksson I, Thunberg U, Johnson A, Backlin C, Enblad G, Sundstrom C, Roos G, Rosenquist R: Somatic hypermutation and $\mathrm{V}(\mathrm{H})$ gene usage in mantle cell lymphoma. Eur $\mathrm{J}$ Haematol 2002, 68:217-224

12. Walsh SH, Thorselius M, Johnson A, Soderberg O, Jerkeman M, Bjorck E, Eriksson I, Thunberg U, Landgren O, Ehinger M, Lofvenberg E, Wallman K, Enblad G, Sander B, PorwitMacDonald A, Dictor M, Olofsson T, Sundstrom C, Roos G, Rosenquist R: Mutated VH genes and preferential VH3-21 use define new subsets of mantle cell lymphoma. Blood 2003, 101:4047-4054

13. Hadzidimitriou A, Agathangelidis A, Darzentas N, Murray F, DelfauLarue MH, Pedersen LB, Lopez AN, Dagklis A, Rombout P, Beldjord K, Kolstad A, Dreyling MH, Anagnostopoulos A, Tsaftaris A, Mavragani-Tsipidou P, Rosenwald A, Ponzoni M, Groenen P, Ghia P, Sander B, Papadaki T, Campo E, Geisler C, Rosenquist R, Davi F, Pott C, Stamatopoulos K: Is there a role for antigen selection in mantle cell lymphoma? Immunogenetic support from a series of 807 cases. Blood 2011, 118:3088-3095

14. Agathangelidis A, Hadzidimitriou A, Rosenquist R, Stamatopoulos K: Unlocking the secrets of immunoglobulin receptors in mantle cell lymphoma: implications for the origin and selection of the malignant cells. Semin Cancer Biol 2011, 21:299-307

15. Sutton LA, Agathangelidis A, Belessi C, Darzentas N, Davi F, Ghia P, Rosenquist R, Stamatopoulos K: Antigen selection in B-cell lymphomas-tracing the evidence. Semin Cancer Biol 2013, 23:399-409

16. Navarro A, Clot G, Royo C, Jares P, Hadzidimitriou A, Agathangelidis A, Bikos V, Darzentas N, Papadaki T, Salaverria I, Pinyol M, Puig X, Palomero J, Vegliante MC, Amador V, MartinezTrillos A, Stefancikova L, Wiestner A, Wilson W, Pott C, Calasanz MJ, Trim N, Erber W, Sander B, Ott G, Rosenwald A, Colomer D, Gine E, Siebert R, Lopez-Guillermo A, Stamatopoulos K, Bea S, Campo E: Molecular subsets of mantle cell lymphoma defined by the IGHV mutational status and SOX11 expression have distinct biologic and clinical features. Cancer Res 2012, 72:5307-5316

17. Kridel R, Meissner B, Rogic S, Boyle M, Telenius A, Woolcock B, Gunawardana J, Jenkins C, Cochrane C, Ben-Neriah S, Tan K, Morin RD, Opat S, Sehn LH, Connors JM, Marra MA, Weng AP, Steidl C, Gascoyne RD: Whole transcriptome sequencing reveals recurrent NOTCH1 mutations in mantle cell lymphoma. Blood 2012, 119:1963-1971

18. Wang ML, Rule S, Martin P, Goy A, Auer R, Kahl BS, Jurczak W, Advani RH, Romaguera JE, Williams ME, Barrientos JC, Chmielowska E, Radford J, Stilgenbauer S, Dreyling M, Jedrzejczak WW, Johnson P, Spurgeon SE, Li L, Zhang L, Newberry K, Ou Z, Cheng N, Fang B, McGreivy J, Clow F, Buggy JJ, Chang BY, Beaupre DM, Kunkel LA, Blum KA: Targeting BTK with ibrutinib in relapsed or refractory mantlecell lymphoma. N Engl J Med 2013, 369:507-516

19. Swerdlow SH, Campo E, Harris NL, Jaffe ES, Pileri SA, Stein H, Thiele J, Vardiman JW (Eds): WHO Classification of Tumours of Haematopoietic and Lymphoid Tissues. ed 4. WHO Classification of Tumours, vol 2. Lyon, France: IARC Press, 2008

20. Lefranc MP, Giudicelli V, Ginestoux C, Jabado-Michaloud J, Folch G, Bellahcene F, Wu Y, Gemrot E, Brochet X, Lane J, Regnier L, Ehrenmann F, Lefranc G, Duroux P: IMGT, the international ImMunoGeneTics information system. Nucleic Acids Res 2009, 37: D1006-D1012

21. Brochet X, Lefranc MP, Giudicelli V: IMGT/V-QUEST: the highly customized and integrated system for IG and TR standardized V-J and V-D-J sequence analysis. Nucleic Acids Res 2008, 36: W503-W508

22. Sutton LA, Kostareli E, Stalika E, Tsaftaris A, Anagnostopoulos A, Darzentas N, Rosenquist R, Stamatopoulos K: Temporal dynamics of clonal evolution in chronic lymphocytic leukemia with stereotyped
IGHV4-34/IGKV2-30 antigen receptors: longitudinal immunogenetic evidence. Mol Med 2013, 19:230-236

23. Sutton LA, Kostareli E, Hadzidimitriou A, Darzentas N, Tsaftaris A, Anagnostopoulos A, Rosenquist R, Stamatopoulos K: Extensive intraclonal diversification in a subgroup of chronic lymphocytic leukemia patients with stereotyped IGHV4-34 receptors: implications for ongoing interactions with antigen. Blood 2009, 114:4460-4468

24. Kostareli E, Sutton LA, Hadzidimitriou A, Darzentas N, Kouvatsi A, Tsaftaris A, Anagnostopoulos A, Rosenquist R, Stamatopoulos K: Intraclonal diversification of immunoglobulin light chains in a subset of chronic lymphocytic leukemia alludes to antigen-driven clonal evolution. Leukemia 2010, 24:1317-1324

25. Marantidou F, Dagklis A, Stalika E, Korkolopoulou P, Saetta A, Anagnostopoulos A, Laoutaris N, Stamatopoulos K, Belessi C, Scouras Z, Patsouris E: Activation-induced cytidine deaminase splicing patterns in chronic lymphocytic leukemia. Blood Cells Mol Dis 2010, 44:262-267

26. Damle RN, Wasil T, Fais F, Ghiotto F, Valetto A, Allen SL, Buchbinder A, Budman D, Dittmar K, Kolitz J, Lichtman SM, Schulman P, Vinciguerra VP, Rai KR, Ferrarini M, Chiorazzi N: Ig V gene mutation status and CD38 expression as novel prognostic indicators in chronic lymphocytic leukemia. Blood 1999, 94:1840-1847

27. Hamblin TJ, Davis Z, Gardiner A, Oscier DG, Stevenson FK: Unmutated Ig $\mathrm{V}(\mathrm{H})$ genes are associated with a more aggressive form of chronic lymphocytic leukemia. Blood 1999, 94:1848-1854

28. Murray F, Darzentas N, Hadzidimitriou A, Tobin G, Boudjogra M, Scielzo C, Laoutaris N, Karlsson K, Baran-Marzsak F, Tsaftaris A, Moreno C, Anagnostopoulos A, Caligaris-Cappio F, Vaur D, Ouzounis C, Belessi C, Ghia P, Davi F, Rosenquist R, Stamatopoulos K: Stereotyped patterns of somatic hypermutation in subsets of patients with chronic lymphocytic leukemia: implications for the role of antigen selection in leukemogenesis. Blood 2008, 111: $1524-1533$

29. Bikos V, Darzentas N, Hadzidimitriou A, Davis Z, Hockley S, Traverse-Glehen A, Algara P, Santoro A, Gonzalez D, Mollejo M, Dagklis A, Gangemi F, Bosler DS, Bourikas G, Anagnostopoulos A, Tsaftaris A, Iannitto E, Ponzoni M, Felman P, Berger F, Belessi C, Ghia P, Papadaki T, Dogan A, Degano M, Matutes E, Piris MA, Oscier D, Stamatopoulos K: Over 30\% of patients with splenic marginal zone lymphoma express the same immunoglobulin heavy variable gene: ontogenetic implications. Leukemia 2012, 26:1638-1646

30. Babbage G, Garand R, Robillard N, Zojer N, Stevenson FK, Sahota SS: Mantle cell lymphoma with $\mathrm{t}(11 ; 14)$ and unmutated or mutated VH genes expresses AID and undergoes isotype switch events. Blood 2004, 103:2795-2798

31. Guikema JE, Rosati S, Akkermans K, Bende RJ, van Noesel CJ, van Krieken JH, Hansmann ML, Schuuring E, Kluin PM: Quantitative RT-PCR analysis of activation-induced cytidine deaminase expression in tissue samples from mantle cell lymphoma and B-cell chronic lymphocytic leukemia patients. Blood 2005, 105:2997-2998. discussion 8-9

32. Klapper W, Szczepanowski M, Heidorn K, Muschen M, Liedtke S, Sotnikova A, Andersen NS, Greeve J, Parwaresch R: Immunoglobulin class-switch recombination occurs in mantle cell lymphomas. J Pathol 2006, 209:250-257

33. Herishanu Y, Perez-Galan P, Liu D, Biancotto A, Pittaluga S, Vire B, Gibellini F, Njuguna N, Lee E, Stennett L, Raghavachari N, Liu P, McCoy JP, Raffeld M, Stetler-Stevenson M, Yuan C, Sherry R, Arthur DC, Maric I, White T, Marti GE, Munson P, Wilson WH, Wiestner A: The lymph node microenvironment promotes B-cell receptor signaling, NF-kappaB activation, and tumor proliferation in chronic lymphocytic leukemia. Blood 2011, 117:563-574

34. Ntoufa S, Vardi A, Papakonstantinou N, Anagnostopoulos A, Aleporou-Marinou V, Belessi C, Ghia P, Caligaris-Cappio F, Muzio M, Stamatopoulos K: Distinct innate immunity pathways to activation and tolerance in subgroups of chronic lymphocytic leukemia with distinct immunoglobulin receptors. Mol Med 2012, 18:1281-1291 
35. Bomben R, Gobessi S, Dal Bo M, Volinia S, Marconi D, Tissino E, Benedetti D, Zucchetto A, Rossi D, Gaidano G, Del Poeta G, Laurenti L, Efremov DG, Gattei V: The miR-17 approximately 92 family regulates the response to Toll-like receptor 9 triggering of CLL cells with unmutated IGHV genes. Leukemia 2012, 26: 1584-1593

36. Saba N, Wiestner A: Do mantle cell lymphomas have an 'Achilles heel'? Curr Opin Hematol 2014, 21:350-357

37. Shinkura R, Ito $S$, Begum NA, Nagaoka $H$, Muramatsu $M$, Kinoshita K, Sakakibara Y, Hijikata H, Honjo T: Separate domains of AID are required for somatic hypermutation and class-switch recombination. Nat Immunol 2004, 5:707-712

38. Wu X, Darce JR, Chang SK, Nowakowski GS, Jelinek DF: Alternative splicing regulates activation-induced cytidine deaminase (AID): implications for suppression of AID mutagenic activity in normal and malignant B cells. Blood 2008, 112:4675-4682
39. van Maldegem F, Scheeren FA, Aarti Jibodh R, Bende RJ, Jacobs H, van Noesel CJ: AID splice variants lack deaminase activity. Blood 2009, 113:1862-1864; author reply 4

40. Albesiano E, Messmer BT, Damle RN, Allen SL, Rai KR, Chiorazzi N: Activation-induced cytidine deaminase in chronic lymphocytic leukemia B cells: expression as multiple forms in a dynamic, variably sized fraction of the clone. Blood 2003, 102:3333-3339

41. McCarthy H, Wierda WG, Barron LL, Cromwell CC, Wang J, Coombes KR, Rangel R, Elenitoba-Johnson KS, Keating MJ, Abruzzo LV: High expression of activation-induced cytidine deaminase (AID) and splice variants is a distinctive feature of poor-prognosis chronic lymphocytic leukemia. Blood 2003, 101:4903-4908

42. Oppezzo P, Vuillier F, Vasconcelos Y, Dumas G, Magnac C, PayelleBrogard B, Pritsch O, Dighiero G: Chronic lymphocytic leukemia B cells expressing AID display dissociation between class switch recombination and somatic hypermutation. Blood 2003, 101:4029-4032 